\title{
On the group of automorphisms of a quasi-affine variety
}

\author{
Zbigniew Jelonek
}

Received: 8 March 2014 / Revised: 19 July 2014 / Published online: 16 November 2014

(C) The Author(s) 2014. This article is published with open access at Springerlink.com

\begin{abstract}
Let $\mathbb{K}$ be an algebraically closed field of characteristic zero. We show that if the automorphisms group of a quasi-affine variety $X$ over $\mathbb{K}$ is infinite, then $X$ is uniruled.
\end{abstract}

Mathematics Subject Classification 14 R 10

\section{Introduction}

Automorphism groups of open varieties have always attracted a lot of attention, but the nature of these groups is still not well-known. For example the group of automorphisms of $\mathbb{K}^{n}$ is understood only in the case $n=2$ (and $n=1$, of course). Let $Y$ be an open variety. It is natural to ask when the group $\operatorname{Aut}(Y)$ of automorphisms of $Y$ is finite. A partial answer to this question is given in our papers [7-9] and [10]. In [6], Iitaka proved that $\operatorname{Aut}(Y)$ is finite if $Y$ has a maximal logarithmic Kodaira dimension. Here we focus on the group of automorphisms of an affine or, more generally, quasi-affine variety over an algebraically closed field of characteristic zero. Let us recall that a quasi-affine variety is an open subvariety of some affine variety. We prove the following:

Theorem 1.1 Let $X$ be a quasi-affine (in particular affine) variety over an algebraically closed field of characteristic zero. If the automorphism group $\operatorname{Aut}(X)$ is infinite, then $X$ is uniruled, i.e., $X$ is covered by rational curves.

The author was partially supported by the Grant of NCN, no. 2013/09/B/ST1/04162, 2014-2017.

Z. Jelonek (凶)

Instytut Matematyczny, Polska Akademia Nauk, Śniadeckich 8, Warszaw 00-956, Poland

e-mail: najelone@cyf-kr.edu.pl 
This generalizes our old results from [9] and [10]. Our proof uses in a significant way a recent progress in the Minimal Model Program (see $[1,2,14]$ ) and is based on our old ideas from [7-9] and [10].

In particular, if $X$ is a quasi-affine non-uniruled variety, then the automorphism group $\operatorname{Aut}(X)$ of $X$ is finite. We show (cf. Proposition 7.2) that conversely, for every $k \geq 1$ and every finite group $G$ there is a $k$-dimensional affine (smooth) non-uniruled variety $X_{G}^{k}$ such that $\operatorname{Aut}\left(X_{G}^{k}\right)=G$. Hence in this version our result is optimal.

If a variety $X$ is uniruled it may happened that the group $\operatorname{Aut}(X)$ is infinite and discrete. Indeed, M. H, El-Huti [3] showed the following interesting fact:

Example 1.2 Take the cubic surface $H_{c} \subset \mathbb{C}^{3}$ defined by $x^{2}+y^{2}+z^{2}-x y z=c, c \in$ $\mathbb{C}$. Then the group $\operatorname{Aut}\left(H_{c}\right)$ is generated by a subgroup $G$ isomorphic to $\mathbb{Z} / 2 \star \mathbb{Z} / 2 \star \mathbb{Z} / 2$ and a finite subgroup $V$ induced by affine linear mappings that preserves $H_{c}$. In fact

$$
\operatorname{Aut}\left(H_{c}\right)=(\mathbb{Z} / 2 \star \mathbb{Z} / 2 \star \mathbb{Z} / 2) \rtimes S_{4},
$$

where $S_{4}$ is the permutation group in 4 elements, see [11].

However, it is possible that if $\operatorname{Aut}(X)$ is non-discrete, we can obtain a more precise information on $X$ than merely non-uniruledness. In particular Hanspeter Kraft and Mikhail Zaidenberg proposed the following:

Kraft-Zaidenberg Conjecture. Assume that $X$ is a quasi-affine variety with nondiscrete group of automorphisms. Than on $X$ acts effectively either the group $\mathbb{G}_{a}=$ $\{\mathbb{K}, 0,+\}$ or the group $\mathbb{G}_{m}=\left\{\mathbb{K}^{*}, 1, \cdot\right\}$.

\section{Terminology}

We assume that the ground field $\mathbb{K}$ is algebraically closed of characteristic zero. For an algebraic variety $X$ (variety is here always irreducible) we denote by $\operatorname{Aut}(X)$ the group of all regular automorphisms of $X$ and by $\operatorname{Bir}(X)$ the group of all birational transformations of $X$. By $\operatorname{Aut}_{1}(X)$ we mean the group of all birational transformation which are regular in codimension one, i.e., which are regular isomorphisms outside subsets of codimension at least two. If $X \subset \mathbb{P}^{n}(\mathbb{K})$ then we put $\operatorname{Lin}(X)=\{f \in$ $\left.\operatorname{Aut}(X): f=\operatorname{res}_{X} T, T \in \operatorname{Aut}\left(\mathbb{P}^{n}(\mathbb{K})\right)\right\}$. Of course, the group $\operatorname{Lin}(X)$ is always an affine group.

Let $f: X-\rightarrow Y$ be a rational mapping between projective normal varieties. Then $f$ is determined outside some (minimal) closed subset $F$ of codimension at least two. If $S \subset X$ and $S \not \subset F$ then by $f(S)$ we mean the set $f(S \backslash F)$. Similarly for $R \subset Y$ we will denote the set $\{x \in X \backslash F: f(x) \in R\}$ by $f^{-1}(R)$.

If $f: X-\rightarrow Y$ is a birational mapping and the mapping $f^{-1}$ does not contract any divisor, we say that $f$ is a birational contraction.

An algebraic variety $X$ of dimension $n>0$ is called uniruled if there exists a variety $W$ of dimension $n-1$ and a rational dominant mapping $\phi: W \times \mathbb{P}^{1}(\mathbb{K})-\rightarrow X$. Equivalently, an algebraic variety $X$ is uniruled if and only if for every point $x \in X$, there exists a rational curve $\Gamma_{x}$ in $X$ through this point. 
We say that a divisor $D$ is $\mathbb{Q}$-Cartier if for some non-zero integer $m \in \mathbb{Z}$ the divisor $m D$ is Cartier. If every divisor on $X$ is $\mathbb{Q}$-Cartier, then we say that $X$ is $\mathbb{Q}$-factorial.

In this paper we treat a hypersurface $H=\bigcup_{i=1}^{r} H_{i} \subset X$ as a reduced divisor $\sum_{i=1}^{r} H_{i}$, and conversly a reduced divisor will be treated as a hyperserface.

\section{Weil divisors on a normal variety}

In this section we recall (with suitable modifications) some basic results about divisors on a normal variety (see e.g., [5]).

Definition 3.1 Let $X$ be a normal complete variety. We will denote by $\operatorname{Div}(X)$ the group of all Weil divisors on $X$. For $D \in \operatorname{Div}(X)$ the set of all effective Weil divisors linearly equivalent to $D$ is called a complete linear system given by $D$ and denoted by $|D|$. Moreover, we set $L(D):=\{f \in \mathbb{K}(X): f=0$ or $D+(f) \geq 0\}$.

We have the following (e.g., [5], 2.16, p.126)

Proposition 3.2 If $D$ is an effective divisor on a normal complete variety $X$, then $L(D)$ is a finite-dimensional vector space (over $\mathbb{K}$ ).

Remark 3.3 The set $|D|$ (if non-empty) has a natural structure of projective space of dimension $\operatorname{dim} L(D)-1$. By a basis of $|D|$ we mean any subset $\left\{D_{0}, \ldots, D_{n}\right\} \subset|D|$ such that $D_{i}=D+\left(\phi_{i}\right)$ and $\left\{\phi_{0}, \ldots, \phi_{n}\right\}$ is a basis of $L(D)$.

Let us recall the next

Definition 3.4 If $D$ is an effective Weil divisor on a normal complete variety $X$, then by a canonical mapping given by $|D|$ and a basis $\phi$ we mean the mapping $i_{(D, \phi)}=$ $\left(\phi_{0}: \ldots: \phi_{n}\right): X \rightarrow \mathbb{P}^{n}(\mathbb{K})$, where $\phi=\left\{\phi_{0}, \ldots, \phi_{n}\right\} \subset L(D)$ is a basis of $L(D)$.

Let $X$ be a normal variety and $Z$ a closed subvariety of $X$. Put $X^{\prime}=X \backslash Z$. We would like to compare the groups $\operatorname{Div}(X)$ and $\operatorname{Div}\left(X^{\prime}\right)$. It can be easily checked that the following proposition is true (compare [4], 6.5., p. 133):

Proposition 3.5 Let $j_{X^{\prime}}: \operatorname{Div}(X) \ni \sum_{i=1}^{r} n_{i} D_{i} \rightarrow \sum_{i=1}^{r} n_{i}\left(D_{i} \cap X^{\prime}\right) \in \operatorname{Div}\left(X^{\prime}\right)$. Then $j_{X^{\prime}}$ is an epimorphism that preserves linear equivalence. If additionally $\operatorname{codim} Z \geq 2$, then $j_{X^{\prime}}$ is an isomorphism.

Now we define the pull-back of a divisor under a rational map $f: X-\rightarrow Y$. Recall that a Cartier divisor can be given by a system $\left\{U_{\alpha}, \phi_{\alpha}\right\}$, where $\left\{U_{\alpha}\right\}$ is some open covering of $X, \phi_{\alpha} \in \mathcal{O}\left(U_{\alpha}\right)$ and $\phi_{\alpha} / \phi_{\beta} \in \mathcal{O}^{*}\left(U_{\alpha} \cap U_{\beta}\right)$.

Definition 3.6 Let $f: X \rightarrow Y$ be a dominant morphism between complete varieties. Let $D$ be a Cartier divisor on $Y$ given by a system $\left\{U_{\alpha}, \phi_{\alpha}\right\}$. By the pullback of the divisor $D$ by $f$ we mean the divisor $f^{*} D$ given by the system $\left\{f^{-1}\left(U_{\alpha}\right), \phi_{\alpha} \circ f\right\}$. More generally if $X, Y$ are complete and let $f$ be a rational map. If $X_{f}$ denotes the domain of $f$, we put $f^{*}(D):=\left(j_{X_{f}}\right)^{-1}\left(\operatorname{res}_{X_{f}} f\right)^{*} D$. Finally let $f$ be as above and let $D$ be an arbitrary Weil divisor on $Y$. Let us assume additionally that codim $f^{-1}(\operatorname{Sing}(Y)) \geq 2$. Then we have a regular map $f: X_{f} \backslash W \rightarrow Y_{\text {reg }}$ (where $W:=f^{-1}(\operatorname{Sing}(Y))$ and we put $f^{*} D:=\left(j_{X_{f} \backslash W}\right)^{-1} f^{*}\left(j_{Y_{\text {reg }}}(D)\right)$. 
By a simple verification we have:

Proposition 3.7 Let $f: X-\rightarrow Y$ be a dominant rational mapping between complete normal varieties, such that $f^{-1}(\operatorname{Sing}(Y))$ has codimension at least two. Then $f^{*}$ : $\operatorname{Div}(Y) \ni D \rightarrow f^{*} D \in \operatorname{Div}(X)$ is a well-defined homomorphism preserving linear equivalence. Moreover, $\operatorname{Supp}\left(f^{*}(D)\right)$ coincides with the $\operatorname{dim} X-1$-dimensional part of the set $\overline{f^{-1}(\operatorname{Supp}(D))}$. In particular if $D$ is an effective Cartier divisor, we have $\operatorname{Supp}\left(f^{*}(D)\right)=\overline{f^{-1}(\operatorname{Supp}(D))}$.

Proof Let $W:=\overline{f^{-1}(\operatorname{Sing}(Y))}$. By the assumption we have codim $W \geq 2$. Take $X^{\prime}:=X_{f} \backslash W$. Since $X$ and $X^{\prime}$ differ by subsets of codimension at least two it is enough to prove our statement for regular mapping $f^{\prime}: X^{\prime} \rightarrow Y$ and for Weil divisors with support outside $\operatorname{Sing}(Y)$, i.e., for Cartier divisors on a smooth variety. But now the statement is obvious.

Corollary 3.8 Let $f$ be as in Proposition 3.7. Let us assume additionally that $f$ is an isomorphism in codimension one. Then $f^{*}: \operatorname{Div}(Y) \rightarrow \operatorname{Div}(X)$ is an isomorphism preserving linear equivalence.

Finally we have the following important result:

Proposition 3.9 Let $X$ be a normal complete variety and $f \in \operatorname{Aut}_{1}(X)$. Let $D$ be an effective divisor on $X$ and $f^{*} D^{\prime}=D$. Then $\operatorname{dim}|D|=\operatorname{dim}\left|D^{\prime}\right|:=n$ and there exists a unique automorphism $T(f) \in \operatorname{Aut}\left(\mathbb{P}^{n}(\mathbb{K})\right)$ such that the folowing diagram commutes

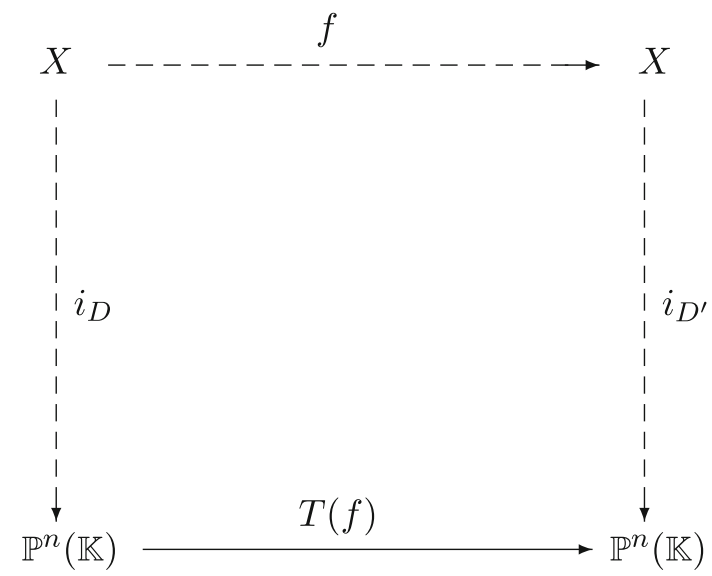

Proof First of all let us note that $T(f)$, if it exists, is unique. Further, by Corollary 3.8, we have $f^{*}\left(\left|D^{\prime}\right|\right)=|D|$ and $f^{*}$ transforms any basis of $\left|D^{\prime}\right|$ onto a basis of $|D|$. Let $\phi$ and $\psi$ be suitable bases such that $i_{D}=i_{(D, \phi)}$ and $i_{D^{\prime}}=i_{\left(D^{\prime}, \psi\right)}$.

We have $i_{D^{\prime}} \circ f=\left(\psi_{0}, \ldots, \psi_{n}\right) \circ f$. But $f^{*}\left(D^{\prime}+\left(\psi_{i}\right)\right)=f^{*}\left(D^{\prime}\right)+f^{*}\left(\psi_{i}\right)=$ $D+\left(\psi_{i} \circ f\right)$. It means that rational functions $\left(\psi_{i} \circ f\right), i=0, \ldots, n$ form a basis of $L(D)$. Hence there exists a non-singular matrix $\left[a_{i j}\right]$ such that $\psi_{i} \circ f=\sum_{j=0}^{n} a_{i j} \phi_{j}$. Now it is clear that it is enough to take as $T(f)$ the projective automorphism of $\mathbb{P}^{n}(\mathbb{K})$ given by the matrix $\left[a_{i j}\right]$. 
Corollary 3.10 Let $G$ be a subgroup of $\operatorname{Aut}_{1}(X)$ such that $G^{*} D=D$ for some effective divisor $D$. Let us denote $\overline{i_{D}(X)}=X^{\prime} \subset \mathbb{P}^{n}(\mathbb{K}), n=\operatorname{dim}|D|$. Then there is a natural homomorphism $T: G \rightarrow \operatorname{Lin}\left(X^{\prime}\right)$. Moreover, if $D$ is very big (i.e., the mapping $i_{D}$ is a birational embedding), then $T$ is a monomorphism.

Proof It is enough to take above $D^{\prime}=D$ and $\phi=\psi$. The last statement is obvious.

Remark 3.11 In our application we deal only with normal $\mathbb{Q}$-factorial varieties. Hence we could restrict our attention only to $\mathbb{Q}$-Cartier divisors. However, the author thinks that the language of Weil divisors is more natural here.

\section{Varieties with good covers}

We begin this section by recalling the definition of a big divisor ( see [12], p. 67):

Definition 4.1 Let $X$ be a projective $n$-dimensional variety and $D$ a Cartier divisor on $X$. The divisor $D$ is called big if $\operatorname{dim} H^{0}\left(X, \mathcal{O}_{X}(k D)\right)>c k^{n}$ for some $c>0$ and $k>>1$.

If $f: X \rightarrow Y$ is a birational morphisms and $D$ is a big (Cartier) divisor, then its pullback $f^{*}(D)$ is also big. Indeed, the line bundle $\mathcal{O}_{X}\left(m f^{*}(D)\right)=f^{*} \mathcal{O}_{Y}(m D)$ has at least as many sections as the bundle $\mathcal{O}_{Y}(m D)$. We show later that it is also true for suitable birational mappings (see Lemma 4.5). We have the following characterization of big divisors (see [12], Lemma 2.60, p. 67):

Proposition 4.2 Let $X$ be a projective $n$-dimensional variety and $D$ a Cartier divisor on $X$. Then the following are equivalent:

1. $D$ is big,

2. for some $m \geq 1$ we have $m D \sim A+E$, where $A$ is ample and $E$ is effective Cartier divisor,

3. for $m>>0$ the rational map $\iota_{m D}$ associated with the system $|m D|$ is a birational embedding,

4. the image of $\iota_{m D}$ has dimension $n$ for $m>>0$.

In the sequel we need the following observation:

Lemma 4.3 Let $X$ be a smooth projective variety and let $D=\sum_{i=1}^{r} a_{i} D_{i}$ be a big divisor on $X$. Then $\operatorname{Supp}(D)=\sum_{i=1}^{r} D_{i}$ is also a big divisor on $X$.

Proof Let $a=\max _{i=1, \ldots, r}\left\{a_{i}\right\}$ and $b_{i}=a-a_{i}$. The divisor $E=\sum_{i=1}^{r} b_{i} D_{i}$ is effective. By condition 2) of Proposition 4.2 the divisor $D+E=a \operatorname{Supp}(D)$ is also big. Hence we conclude by 3 ) of Proposition 4.2.

Definition 4.4 Let $X$ be a normal projective variety and let $D$ be a Weil divisor on $X$. We say that $D$ is very big if the rational map $\iota_{D}$ associated with the system $|D|$ is a birational embedding. We say that $D$ is big if for some $m \geq 1$ the divisor $m D$ is very big. 
It is easy to see that for Cartier divisors this definition coincide with the previous one. We have the following simple lemma:

Lemma 4.5 Let $X, Y$ be normal projective varieties and let $\phi: X-\rightarrow Y$ be a birational mapping such that $\operatorname{codim} \phi^{-1}(\operatorname{Sing}(Y)) \geq 2$. If $D$ is an effective big divisor on $Y$, then the divisor $\phi^{*}(D)$ on $X$ is also big.

Proof It is enough to assume that $D$ is very big and prove that then $\phi^{*}(D)$ is also very big. Take $f_{0}=1$ and let divisors $\left\{D+\left(f_{0}\right), D+\left(f_{1}\right), \ldots, D+\left(f_{s}\right)\right\}$, where the $f_{i} \in \mathbb{K}(Y)$, form a basis of the system $|D|$. By the assumption, the regular mapping $\Psi: Y \backslash \operatorname{Supp}(D) \ni x \mapsto\left(f_{1}(x), \ldots, f_{s}(x)\right) \in \mathbb{K}^{s}$ is a birational morphism. The system $\left|\phi^{*}(D)\right|$ contains divisors $\left\{\phi^{*}(D), \phi^{*}(D)+\left(f_{1} \circ \phi\right), \ldots, \phi^{*}(D)+\left(f_{s} \circ \phi\right)\right\}$. Since the collection of rational functions $1, f_{1} \circ \phi, \ldots, f_{s} \circ \phi$ is linearly independent, we can extend it to some basis $B$ of $L\left(\phi^{*}(D)\right)$. Let $\Psi^{\prime}: X \backslash\left|\operatorname{Supp}\left(\phi^{*}(D)\right)\right| \rightarrow \mathbb{K}^{N}$ be a mapping given by a system $\left|\phi^{*}(D)\right|$ and the basis $B$. The mapping $\Psi^{\prime}$ composed with a suitable projection $\mathbb{K}^{N} \rightarrow \mathbb{K}^{s}$ is equal to $\Psi \circ \phi$. Since the latter mapping is birational, the mapping $\Psi^{\prime}$ is also birational.

We shall use:

Definition 4.6 Let $X$ be an (open) variety. We say that $X$ has a good cover $Y$, if there exists a completion $\bar{X}$ of $X$ and a smooth projective variety $Y$ with a birational morphism $g: Y \rightarrow \bar{X}$ such that:

1. $D:=g^{-1}(\bar{X} \backslash X)$ is a big hypersurface in $Y$,

2. $\operatorname{Aut}(X) \subset \operatorname{Aut}(Y \backslash D)$, i.e., every automorphism of $X$ can be lifted to an automorphism of $Y \backslash D$.

Our next aim is to show that quasi-affine varieties have good covers.

Proposition 4.7 Any quasi-affine variety $X$ has a good cover.

Proof By the assumption, there is an affine variety $X_{1}$ such that $X \subset X_{1}$ is an open dense subset. Since $X_{1}$ is affine, we can assume that it is a closed subvariety of some $\mathbb{K}^{N}$. Denote by $\bar{X}$ the projective closure of $X_{1}$ in $\mathbb{P}^{N}$. Let $\pi_{\infty}$ be the hyperplane at infinity in $\mathbb{P}^{N}$ and $V:=\bar{X} . \pi_{\infty}$ be a divisor at infinity on $\bar{X}$. Of course $V$ is a big (even very ample) Cartier divisor.

Let $h: Y \rightarrow \bar{X}$ be a canonical desingularization of $\bar{X}$ ( see e.g., [13,16]). Then $h_{\mid h-1(X)}: h^{-1}(X) \rightarrow X$ is a canonical desingularization of $X$. In particular every automorphism of $X$ has a lift to an automorphism of $h^{-1}(X)$, i.e., $\operatorname{Aut}(X) \subset \operatorname{Aut}\left(h^{-1}(X)\right)=\operatorname{Aut}\left(Y \backslash h^{-1}(V)\right)$. Since $V$ is a big divisor, so is its pullback $h^{*}(V)$.

Note that $Z:=Y \backslash h^{-1}(X)$ is a closed subvariety of $Y$. Let $J_{Z}$ be the ideal sheaf of $Z$ and let $f: Y^{\prime} \rightarrow Y$ be a canonical principalization of $J_{Z}$ (see e.g., $\left.[13,16]\right)$. Thus $D:=$ $f^{-1}(Z)$ is a hypersurface, which contains a big hypersurface $V^{\prime}=\operatorname{Supp}\left(f^{*} h^{*}(V)\right)$. Since $D=V^{\prime}+E$, where $E$ is an effective divisor, the hypersurface $D$ is also big by Proposition 4.2.

Finally if we take $g=f \circ h: Y^{\prime} \rightarrow \bar{X}$, then conditions 1) and 2) of Definition 4.6 are satisfied. 


\section{The Quasi minimal model}

In this section, following [14], we introduce the notion of quasi-minimal models (for details see [14]). This is a weaker analog of the usual notion of minimal model, which has an advantage that to prove its existence we do not need the full strength of the Minimal Model Program.

Definition 5.1 (See [14]) An effective $\mathbb{Q}$-divisor $M$ on a variety $X$ is said to be $\mathbb{Q}$ movable if for some $n>0$ the divisor $n M$ is integral and generates a linear system without fixed components. Let $\mathrm{X}$ be a projective variety with $\mathbb{Q}$-factorial terminal singularities. We say that $\mathrm{X}$ is a quasi-minimal model if there exists a sequence of $\mathbb{Q}$-movable $\mathbb{Q}$-divisors $M_{j}$ whose limit in the Neron-Severi space $N S W_{\mathbb{Q}}(X)=N S W(X) \otimes \mathbb{Q}$ is $K_{X}$.

By the recent progress in the minimal model program ( see $[1,2,14]$ ), every nonuniruled smooth variety has a quasi-minimal model. In fact, if we ran MMP on $X$ and we do all possible divisorial contractions (and all necessary flips) we achieve a quasi-minimal model $Y$, together with a mapping $\phi: X-\rightarrow Y$ that is a composition of divisorial contractions and flips. In particular $\phi$ is a birational contraction, i.e., the mapping $\phi^{-1}$ does not contract any divisor (cf. [14], section 4, Corollary 4.5). Thus we get:

Theorem 5.2 Let $X$ be a smooth projective non-uniruled variety. Then there is a quasi-minimal model $Y$ and a birational contraction $\phi: X-\rightarrow Y$.

Quasi minimal models have the following very important property (cf. [14], section 4, Proposition 4.6):

Theorem 5.3 Let $X$ be a quasi-minimal model. Then $\operatorname{Bir}(X)=\operatorname{Aut}_{1}(X)$.

\section{Main result}

Now we can start our proof. The first step is

Proposition 6.1 Let $X$ be a normal complete non-uniruled variety and let $H$ be a big hypersurface in $X$. Then the group $\operatorname{Stab}_{X}(H)=\left\{f \in \operatorname{Aut}_{1}(X): f^{*} H=H\right\}$ is finite.

Proof For some $m \in \mathbb{N}$ the divisor $m H$ is very big. We have $\operatorname{Stab}_{X}(H)=\{f \in$ $\left.\operatorname{Aut}_{1}(X): f^{*} H=H\right\}=\operatorname{Stab}_{X}(m H)=\left\{f \in \operatorname{Aut}_{1}(X): f^{*}(m H)=m H\right\}$. By the assumption, the variety $X^{\prime}=\overline{i_{m H}(X)}$ is birationally equivalent to $X$. In view of Corollary 3.10 it is enough to prove that the group $\operatorname{Lin}\left(X^{\prime}\right)$ is finite. Since $X$ is non-uniruled, the variety $X^{\prime}$ is non-uniruled too. But the group $\operatorname{Lin}\left(X^{\prime}\right)$ is an affine group and if it is infinite, then by Rosenlicht Theorem (see [15]), we have that $X^{\prime}$ is ruled - which is impossible.

Now we can prove our main result:

Theorem 6.2 Let $X$ be an open variety with a good cover. If the group $\operatorname{Aut}(X)$ is infinite, then $X$ is uniruled. 
Proof Assume that Aut $(X)$ is infinite. Let $f: \bar{Y} \rightarrow \bar{X}$ be a good cover of $X$ and take $Y=f^{-1}(X)$. Then $\operatorname{Aut}(Y)$ is also infinite. We have to prove that $X$ is uniruled. To do this it suffices to prove that $Y$ is uniruled.

Assume that $Y$ is not uniruled. By Theorem 5.3 there exists a quasi-minimal model $Z$ and a birational contraction $\phi: \bar{Y}-\rightarrow Z$. Take $\psi=\phi^{-1}$. The mapping $\psi$ is a regular mapping outside some closed subset $F$ of codimension $\geq 2$. By the Zariski Main Theorem the mapping $\psi$ restricted to $Z \backslash F$ is an embedding.

Take a mapping $G \in \operatorname{Aut}(Y)$, in fact $G \in \operatorname{Bir}(\bar{Y})$. The mapping $G$ induces a birational mapping $g \in \operatorname{Bir}(Z)$. Since $\operatorname{Bir}(Z)=\operatorname{Aut}_{1}(Z)$ we have $g \in \operatorname{Aut}_{1}(Z)$. The mapping $g$ is a morphisms outside a closed subset $R$ of codimension $\geq 2$. Since the mapping $g$ is an automorphism in codimension one we have codim $\overline{g^{-1}(F)} \geq 2$. Denote $V:=Z \backslash\left(F \cup \overline{g^{-1}(F)} \cup R\right)$ and $U=g(V)$. The mapping $g$ restricted to $V$ is an embedding by the Zariski Main theorem. In particular the set $U$ is open and $g: V \rightarrow U$ is an isomorphism. The mapping $\psi$ embeds sets $V$ and $U$ into $Y$. Denote $V^{\prime}:=\psi(V)$ and $U^{\prime}=\psi(U)$. Under this identification, the mapping $g: V \rightarrow U$ corresponds to the mapping $G: V^{\prime} \rightarrow U^{\prime}$. Let $D=\bar{Y} \backslash Y$ be a big hypersurface, as in the definition of a good cover. The hypersurface $D^{\prime}:=\psi^{*}(D)$ is also big ( see Lemma 4.5) and $D^{\prime} \cap V$ corresponds to $D \cap V^{\prime}$. Since $G\left(V^{\prime} \backslash D\right)=U^{\prime} \backslash D$, we have that $g$ transforms irreducible components of $D^{\prime} \cap V$ onto irreducible components of $D^{\prime} \cap U$. In particular $g^{*}\left(D^{\prime}\right)=D^{\prime}$. This means that $\operatorname{Aut}(Y) \subset \operatorname{Stab}_{Z}\left(D^{\prime}\right) \subset \operatorname{Aut}_{1}(Z)$. By Proposition 6.1 this contradicts our assumption.

Corollary 6.3 Let $X$ be a quasi-affine (in particular affine) variety. If the group $\operatorname{Aut}(X)$ is infinite, then $X$ is uniruled.

\section{Automorphisms of affine non-uniruled varieties}

As we know, if $X$ is a quasi-affine non-uniruled variety, then it has a finite automorphism group. We show now that conversely, for every $k \geq 1$ and every finite group $G$, there is a $k$-dimensional affine (smooth) non-uniruled variety $X_{G}^{k}$ such that $\operatorname{Aut}\left(X_{G}^{k}\right)=G$. We start with:

Lemma 7.1 Let $\Gamma_{1}, \ldots, \Gamma_{k}$ be affine curves with $0<g\left(\Gamma_{1}\right)<g\left(\Gamma_{2}\right)<\ldots g\left(\Gamma_{k}\right)$ (here $g(X)$ denotes the genus of a curve $X$ ). Then

$$
\operatorname{Aut}\left(\prod_{i=1}^{k} \Gamma_{i}\right)=\prod_{i=1}^{k} \operatorname{Aut}\left(\Gamma_{i}\right)
$$

Proof We proceed by induction. The case $k=1$ is trivial. Assume $k>1$. Let $\Phi \in \operatorname{Aut}\left(\prod_{i=1}^{k} \Gamma_{i}\right)$. For a point $a \in \prod_{i=2}^{k} \Gamma_{i}$ let $\Gamma_{a}:=\Gamma_{1} \times\{a\}$ and let $\Gamma_{a}^{\prime}:=\Phi\left(\Gamma_{a}\right)$. Since the curve $\Gamma_{a}$ cannot dominate any curve $\Gamma_{i}$ for $i>1$ we have that $\Gamma_{a}^{\prime}=\Gamma_{1} \times \phi(a)$ where $\phi(a) \in H:=\prod_{i=2}^{k} \Gamma_{i}$. Hence $\Phi: \Gamma_{1} \times H \ni(x, a) \mapsto(\psi(x, a), \phi(a)) \in$ $\Gamma_{1} \times H$. For a fixed $a \in H$, the mapping $\psi(x, a): \Gamma_{1} \ni x \mapsto \psi(x, a) \in \Gamma_{1}$ is an automorphism of $\Gamma_{1}$. Since the group $\operatorname{Aut}\left(\Gamma_{1}\right)$ is finite, we have that $\psi(x, H)$ consists of one point, i.e., the mapping $\psi$ does not depend on $a \in H$. In particular, 
$\psi \in \operatorname{Aut}\left(\Gamma_{1}\right)$. The mapping $\phi: H \rightarrow H$ is an automorphism and we conclude the proof by induction.

Now we prove:

Proposition 7.2 For every $k \geq 1$ and every finite group $G$, there is a $k$-dimensional affine (smooth) non-uniruled variety $X_{G}^{k}$ such that $\operatorname{Aut}\left(X_{G}^{k}\right)=G$.

Proof First we assume $k=1$ and we construct a non-rational curve $\Gamma_{1}$ with $\operatorname{Aut}\left(\Gamma_{1}\right)=$ $G$. Since $G$ is a finite group there is a number $n$ such that $G$ is a subgroup of the permutation group $S_{n}$. Consider a mapping

$$
F: \mathbb{K}^{n} \ni x \mapsto\left(s_{1}(x), \ldots, s_{n}(x)\right) \in \mathbb{K}^{n},
$$

where $s_{1}, \ldots, s_{n}$ are all elementary symmetric polynomials of $n$ variables. The group $S_{n}$ acts effectively on general fibers of $F$. By (a variant of) the Bertini Theorem the inverse image of a general hyperplane is again a smooth irreducible hypersurface (we are in characteristic zero!). If we repeat this argument several times we see that the inverse image $F^{-1}(H)$ of a general plane $H \subset \mathbb{K}^{n}$ is a smooth irreducible surface. Now let $\Lambda$ be a general curve on $H$ of fixed degree $d>2$. Again by the Bertini Theorem the inverse image $\Gamma$ of $\Lambda$ is a smooth irreducible curve. Of course $\Gamma$ is non-rational, in particular it has finite automorphism group and by the construction $S_{n} \subset \operatorname{Aut}(\Gamma)$. Let $x \in \Gamma$ be a general point such that \#Aut $(\Gamma) \cdot x=$ \#Aut $(\Gamma)$. Put $\Gamma_{1}=\Gamma \backslash G . x$. It is easy to see that $\operatorname{Aut}\left(\Gamma_{1}\right)=G$ and we take $X_{G}^{1}:=\Gamma_{1}$. If $k>1$, then we choose curves $\Gamma_{2}, \ldots, \Gamma_{k}$ such that:

1. $\operatorname{Aut}\left(\Gamma_{i}\right)=\{$ identity $\}$,

2. $g\left(\Gamma_{1}\right)<g\left(\Gamma_{2}\right)<\cdots<g\left(\Gamma_{k}\right)$. Now put $X_{G}^{k}:=\prod_{i=1}^{k} \Gamma_{i}$ and apply Lemma 7.1.

Acknowledgments The author is grateful to Professors: Caucher Birkar from the University of Cambridge and Yuri Prokhorov from the Steklov Institute of Mathematics for helpful discussions. The author also warmly thanks the anonymous referee for many helpful comments and the editor for his patience. I would like also to express my gratitude to Professors: Hanspeter Kraft from the University of Basel, Stephane Lamy from the University of Warwick and Serge Cantat from Universite de Rennes I, whose kindly informed me about Example 1.2.

Open Access This article is distributed under the terms of the Creative Commons Attribution License which permits any use, distribution, and reproduction in any medium, provided the original author(s) and the source are credited.

\section{References}

1. Birkar, C.: Existence of log canonical flips and a special LMMP. Publ. Math. Inst. Hautes Etudes Sci. 115, 325-368 (2012)

2. Birkar, C., Cascini, P., Hacon, C., McKernan, J.: Existence of minimal models for varieties of log general type. J. AMS 23(2), 405-468 (2010)

3. El-Huti, M.H.: Cubic surfaces of Markov type. Math. USSR Sbornik 22(3), 333-348 (1974)

4. Hartshorne, R.: Algebraic geometry. Springer, New York (1987)

5. Iitaka, S.: Algebraic geometry. Springer, New York (1982) 
6. Iitaka, S.: On logarithmic Kodaira dimension of algebraic varieties. In: Complex analysis and algebraic geometry, pp. 178-189. Iwanami, Tokyo (1977)

7. Jelonek, Z.: Irreducible identity sets for polynomial automorphisms. Math. Z. 212, 601-617 (1993)

8. Jelonek, Z.: Affine smooth varieties with finite group of automorphisms. Math. Z. 216, 575-591 (1994)

9. Jelonek, Z.: The group of automorphisms of an affine non-uniruled surface. Univ. Iaegel. Acta Math. 32, 65-68 (1995)

10. Jelonek, Z.: The group of automorphisms of an affine non-uniruled variety. Seminari di Geometria, Uniwersita degli Studi di Bologna, pp. 169-180 (1996)

11. Furter, J.P., Kraft, H.: On the geometry of the automorphism group of affine n-space, preprint (2014)

12. Kollar, J., Mori, S.: Birational geometry of algebraic varieties. Cambridge University Press, Cambridge (1998)

13. Kollar, J.: Lectures on Resolution of Singularities. Annals of Mathematics Studies 166 (2007)

14. Prokhorov, Y., Shramov, C.: Jordan property for groups of birational selfmaps. Compositio Math. (to appear). arXiv:1307.1784v2

15. Rosenlicht, M.: Some basic theorems on algebraic groups. Am. J. Math. 78, 401-443 (1956)

16. Włodarczyk, J.: Simple Hironaka resolution in characteristic zero. J. Am. Math. Soc. 18, 779-822 (2005) 